\title{
Unfälle durch Gartenarbeit - Bagatelle oder Ernst?
}

\section{Eine retrospektive Analyse über fünf Jahre im Universitären Notfallzentrum des Inselspitals Bern}

\author{
Accidents Caused by Gardening - Trivial or Serious? 5-Year Retrospective \\ Analysis at the University Emergency Department Berne
}

\author{
Jil Schaudt, Stephan Ziegenhorn, Jasmin Lienert, Aristomenis Exadaktylos \\ und Jolanta Klukowska-Rötzler
}

Universitäres Notfallzentrum, Inselspital, Universitätsspital Bern

\begin{abstract}
Zusammenfassung: Gartenarbeit ist eine beliebte Freizeitaktivität in der Schweiz. Pro Jahr werden ungefähr eine Million Nichtberufsunfälle registriert, davon ereignen sich etwa 600000 zu Hause oder während der Ausübung eines Hobbies, einschliesslich 16000 Unfällen bei der Gartenarbeit. Ziel dieser retrospektiven Analyse ist die Untersuchung von Gartenarbeitsunfällen. Die Daten wurden aus der elektronischen Datenbank des Universitären Notfallzentrums des Inselspitals Bern gesammelt und retrospektiv ausgewertet. Patienten im Alter von 16 Jahren oder älter, die einen Unfall während der Gartenarbeit als Freizeitaktivität erlitten, wurden in die Analyse eingeschlossen. Jüngere Patienten wurden in einem separaten Notfallzentrum für Kinder und Jugendliche behandelt und somit nicht ausgewertet. Anschliessend wurden die Daten nach Alter, Geschlecht, Triagekategorie, Unfallmechanismus, unfallverursachendem Objekt, betroffenen Körperregionen, Diagnosen, Schweregrad der Verletzung (monotraumatisch, kombiniert mit mehr als einer betroffenen Körperregion oder Polytrauma) und ambulanter oder stationärer Behandlung analysiert. Unfälle bei der privaten Gartenarbeit ereigneten sich vorwiegend bei Erwachsenen im Alter von 40-69 Jahren. Dabei waren Männer häufiger als Frauen betroffen. Die Patienten zogen sich vorwiegend Augenverletzungen und Hautwunden zu. Exponierte Körperstellen wie Augen, Finger, Kopf und Gesicht waren am häufigsten betroffen. Es handelte sich vorwiegend um monotraumatische Verletzungen, die ambulant behandelt werden konnten. Stürze und ähnliche Ereignisse wie Stolpern führten häufig zu Unfällen. Unfälle wurden hauptsächlich durch organisches Material oder durch die fehlerhafte und nachlässige Anwendung von Werkzeugen verursacht. Die Datenanalyse zeigte, dass ein Grossteil der Unfälle durch einfache Präventionsmassnahmen verhindert werden könnten.
\end{abstract}

Schlüsselwörter: Gartenarbeit, Unfall, Verletzung, Notfall

\begin{abstract}
Gardening is a popular leisure activity in Switzerland. Approximately 1 million non-occupational accidents are recorded each year; 600,000 of these occur at home or in pursuit of a hobby, including approx. 16,000 accidents while gardening. The aim of this study is to investigate gardening-related accidents. The data for this study were generated from the database of the management system of Berne University Hospital, Switzerland, and retrospectively analyzed. Patients were enrolled who were at least 16 years old - since younger patients in Switzerland are normally treated in specialised Emergency Departments for Paediatrics. The study was restricted to patients who suffered an accident that resulted in physical impairment while working in their own garden. The data were then analysed based on age, sex, triage category, mechanism, causing object, lesion site, diagnoses, severity of injury (monotraumatic or combined with more than one region or polytrauma), in-patient or out-patient treatment. Gardening-related accidents were mainly suffered by middle aged adults (40-69 years). Men were more often affected than women. At consultation, the injury was mostly monotraumatic and could be treated in an out-patient setting. Patients mainly complained of eye injuries and lacerations. The exposed areas of the eyes, fingers and the head or face were the most affected body regions. Falls and stumbling often led to accidents. Injuries were mainly caused by organic material or by the improper use of gardening tools. The data analysis showed that many injuries could have been avoided by simple preventive measures.
\end{abstract}

Keywords: Gardening, emergency, injury, accident

Résumé: Le jardinage est une activité de loisir appréciée en Suisse. Chaque année, notre pays compte un million d'accidents non-professionnels. Environ 600000 accidents se produisent à domicile ou lors de loisirs, dont 16'000 accidents lors d'activités de jardinage. Le but de cette étude rétrospective est l'analyse des accidents de jardinage. Les données ont été rassemblées à partir d'une base de données de l'Hôpital Universitaire de Berne et analysées rétrospectivement. Les patients de 16 
ans et plus ont été inclus, ayant souffert d'un accident avec blessure durant leur activité de jardinage non-professionnel. Les patients de moins de 16 ans sont normalement traités aux urgences pédiatriques spécialisées, raison pour laquelle les données n'ont pas été prises en compte. Par la suite, les données ont été analysées selon l'âge, le sexe, la catégorie de triage, le mécanisme de l'accident, l'objet impliqué dans l'accident, la région du corps concernée, le diagnostic, le degré de gravité des lésions (mono-traumatique, combiné c.à.d. concernant plus d'une partie du corps, ou poly-traumatique), et si la prise en charge était ambulatoire ou nécessitait une hospitalisation. Les accidents de jardinage concernent principalement les personnes entre 40 et 69 ans. Les hommes sont plus affectés que les femmes. Les patients se présentent le plus souvent avec une lésion mono-traumatique, qui peut être prise en charge de manière ambulatoire. Les blessures les plus fréquentes sont des lésions oculaires ou des plaies. Les parties du corps les plus atteintes sont les parties exposées comme les yeux, les doigts, la tête et le visage. Des chutes et trébuchements mènent souvent à des accidents. Les blessures sont principalement causées par du matériel organique ou l'utilisation impropre des outils de jardinage. l'analyse des données démontre que beaucoup de blessures auraient pu être évitées par des mesures de prévention simples.

Mots-clés: Jardinage, accident, blessures, urgences

Gartenarbeit kann positive Auswirkungen auf die Gesundheit von Jung und Alt haben. Verschiedene Studien untersuchten deren Effekt auf Körper, Geist und Wohlbefinden bei Erwachsenen und bestätigen, dass Menschen höheren Alters von Gartenarbeit profitieren [1].

Gärtnern ist eine der häufigsten Freizeitaktivitäten unter Senioren [2]. Gartenarbeit ist auch in der Schweiz ein beliebtes Hobby. Eine Statistik über Freizeitaktivitäten in der Schweiz zeigte, dass 41,1\% der Befragten wöchentlich gärtnern oder heimwerkern, weitere 13,1\% sogar täglich [3].

Pro Jahr werden ungefähr eine Million Nichtberufsunfälle in der Schweiz registriert, davon ereignen sich etwa $600000 \mathrm{zu}$ Hause oder während der Ausübung eines Hobbies, einschliesslich 16000 Unfällen bei der Gartenarbeit. [4]

Unterschiedliche Studien befassten sich mit den Auswirkungen von Gartenarbeit auf Körper, Geist und Wohlbefinden. Zwei Studien zu Augenverletzungen zeigten, dass Gärtnern eine häufige Tätigkeit ist, die mit Augenverletzungen einhergeht $[5,6]$. Ein weiterer Review über den Effekt von Gartenarbeit auf die körperliche Gesundheit und Funktionalität bei Senioren zeigte unterschiedliche Resultate; ein Grossteil der Studien fand keine Korrelation zwischen Gartenarbeit und dem Gesundheitszustand, eine Studie zeigte eine Verbesserung der gesundheitlichen Verfassung, eine weitere eine Beeinträchtigung [7]. Zwei weitere Reviews schlussfolgerten eine Verbesserung der Gesundheit und des Wohlergehens durch Gartenarbeit [8, 9].

Gärtnern führt zu einer nicht vernachlässigbaren Anzahl an Unfällen und weiteren Gesundheits-beeinträchtigungen $[10,11]$. Dennoch sollten die positiven Auswirkungen auf die physische und psychische Gesundheit sowie auf das Wohlergehen nicht vernachlässigt werden [12-14].

Zudem sind Daten zu Nichtberufsunfällen wie Unfälle durch Gärtnern spärlich.

Primäres Ziel dieser retrospektiven Studie ist die Analyse von Gartenarbeitsunfällen, die Erfassung epidemiologischer Daten und die Untersuchung von Unfallursachen, -mechanismen und Folgeschäden. Zudem sollen einfache präventive Massnahmen anhand der erhaltenen Informationen formuliert werden.

\section{Studiendesign und Untersuchungsmethoden}

Das Universitäre Notfallzentrum Inselspital Bern ist die grösste Notfallstation im Kanton Bern und daher Anlaufstelle für Patienten des Kantons und des umliegenden Einzugsgebiets mit schwersten Traumata und Erkrankungen. Das 2012 sanierte Universitäre Notfallzentrum zählt mit seinen drei Hubschrauberlandeplätzen, drei Schockräumen und 30 monitorisierten Kojen zu den modernsten Notfallzentren schweizweit. Im Jahr 2018 wurden mehr als 48000 Patienten an diesem Notfallzentrum behandelt [15].

Die Daten wurden von einem Datenmanager aus der Datenbank des Managementsystems des Universitätsspitals Bern (Ecare, Turnhout, Belgien) zwischen dem 10.05.2012 und dem 27.03.2017 mittels einer Suchabfrage mit den Keywords «Gartenarbeit», «Unfall», «Verletzung» und «Notfall» generiert und anonymisiert.

Die erhaltenen Daten wurden anschliessend mittels der Einschlusskriterien selektioniert. Als erstes wurden lediglich Patienten $\geq 16$ Jahre eingeschlossen, da jüngere Patienten im spezialisierten Notfallzentrum für Kinder und Jugendliche des Inselspitals behandelt wurden und Gartenarbeit hauptsächlich von Erwachsenen praktiziert wird. Als zweites Kriterium fungierte der Unfallbegriff. Als Unfallbegriff nahmen wir die Definition des schweizerischen Bundesgesetzes über den Allgemeinen Teil des Sozialversicherungsrechts. In dieser Definition wird ein Unfall als die plötzliche, nicht beabsichtigte, schädigende Einwirkung eines ungewöhnlichen äusseren Faktors auf den menschlichen Körper beschrieben, die eine Beeinträchtigung der körperlichen, geistigen und psychischen Gesundheit oder den Tod zur Folge hat [16]. Unfallähnliche Körperschädigungen nach dem schweizerischen Bundesgesetz über die Unfallversicherung wurden ebenfalls eingeschlossen [17]. Als drittes Kriterium galt Gartenarbeit im privaten Rahmen; jeder Unfall musste während oder unmittelbar nach dem Gärtnern in der Freizeit erfolgen. Berufsunfälle als Gärtner rufen mutmasslich aufgrund eines anderen Risikoprofils hinsichtlich der Tätigkeiten, Gerätschaften und professionellem Equipment andere Verlet- 
zungen hervor. Zusammenfassend wurden Patienten im Altern von $\geq 16$ Jahren, die einen Unfall während der Gartenarbeit als Freizeitaktivität mit einer resultierenden körperlichen Gesundheitsbeeinträchtigung erlitten, eingeschlossen.

Nachfolgend wurden die selektionierten Daten nach Alter, Geschlecht, Triagekategorie, Unfallmechanismus, unfallverursachendem Objekt, betroffenen Körperregionen, Diagnosen, Schweregrad der Verletzung (monotraumatisch, kombiniert mit mehr als einer betroffenen Körperregion oder Polytrauma) und ambulanter oder stationärer Behandlung analysiert.

Die deskriptive Statistik erfolgte mit Excel.

Die Studie wurde bei der Kantonalen Ethikkommission (KEK) in Bern überprüft und bewilligt. (Ethik Approval KEK-Nummer No 2018-00348). Es wurde keine studienspezifische Einverständniserklärung eingeholt. Die Daten wurden in anonymisierter Form analysiert und publiziert.

\section{Ergebnisse}

Mit den oben beschriebenen Suchkriterien wurden 743 Fälle erfasst. 161 Fälle (21,7\%) erfüllten alle Einschlusskriterien. Sechs Fälle $(0,8 \%)$ wurden aufgrund des Patien-

Gefundene Falldaten mit den beschriebenen Keyword $\mathrm{n}=\mathbf{7 4 3}$

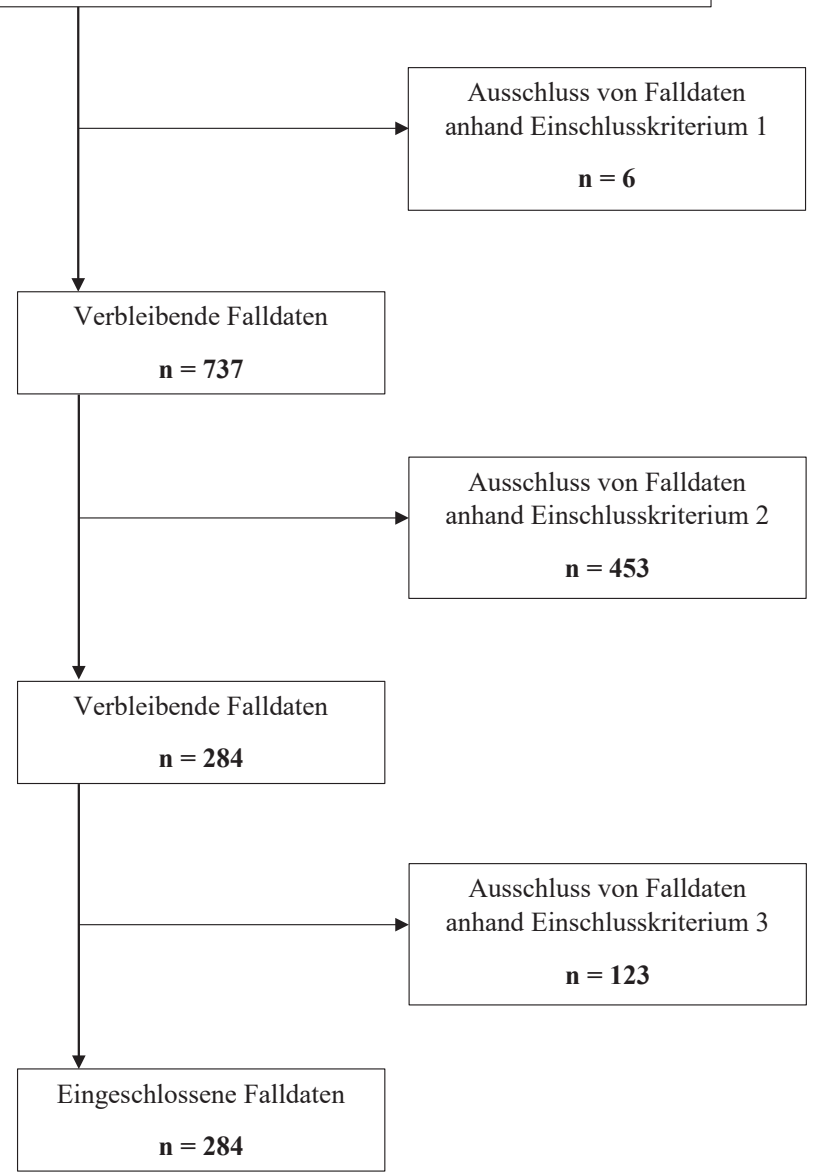

Abbildung 1. Studien-Einschlussdiagramm. tenalters $(<16-j a ̈ h r i g e ~ P a t i e n t e n ~ w u r d e n ~ a u s s c h l i e s s l i c h$ von Pädiatern im Schockraum des Universitären Notfallzentrums behandelt), 453 Fälle (61,0\%) aufgrund des Unfallbegriffs und die restlichen 123 Fälle (16,6\%) aufgrund des Kriteriums der Gartenarbeit als Freizeitaktivität, ausgeschlossen (Abb. 1).

Das Durchschnittsalter der Verunfallten betrug 55,9 Jahre. 57,1 \% der Verunfallten waren Männer, 42,9 \% Frau-

Tabelle 1. Soziodemografische Fakten der Patienten $(n=161)$

\begin{tabular}{lcc}
\hline & Fälle (n) & Prozent (\%) \\
\hline Geschlecht & & \\
\hline Männlich & 92 & 57,1 \\
Weiblich & 69 & 42,9
\end{tabular}

Altersgruppe

\begin{tabular}{lcc}
\hline $16-19$ & 0 & 0,0 \\
$20-29$ & 8 & 5,0 \\
$30-39$ & 15 & 9,3 \\
$40-49$ & 34 & 21,1 \\
$50-59$ & 37 & 23,0 \\
$60-69$ & 36 & 22,4 \\
$70-79$ & 24 & 14,9 \\
$80-89$ & 5 & 3,1 \\
$90-99$ & 2 & 1,2 \\
\hline
\end{tabular}

\begin{tabular}{lcc}
\hline Jahr & & \\
\hline 2012 (Mai-Dezember) & 17 & 10,6 \\
2013 & 37 & 23,0 \\
2014 & 33 & 20,5 \\
2015 & 40 & 24,8 \\
2016 & 26 & 16,1 \\
2017 (Januar-März) & 8 & 5,0
\end{tabular}

Behandlungsart

\begin{tabular}{lcc}
\hline Ambulant & 129 & 80,1 \\
Stationär & 25 & 15,5 \\
Unbekannt & 7 & 4,3
\end{tabular}

Unbekannt

Triagekategorie STS

1 (lebensbedrohlich)

2 (sehr dringend)

13,4

3 (dringend)

19,88

4 (standard)

26,71

5 (nicht dringend)

33,54

Trauma

Monotraumatisch

152

94,4

Kombiniert

3,7

Polytrauma

1,9 
Tabelle 2. Diagnose und betroffene Körperstelle

\begin{tabular}{|c|c|c|c|}
\hline & Monotraumatisch & Kombiniert & Polytraumata \\
\hline \multicolumn{4}{|l|}{ Diagnose } \\
\hline Hautwunde & $51(29,8 \%)$ & $4(22,2 \%)$ & $0(0 \%)$ \\
\hline Weichteilverletzung & $21(12,3 \%)$ & $5(27,8 \%)$ & $1(10,00 \%)$ \\
\hline Fraktur & $15(8,8 \%)$ & $7(38,9 \%)$ & $7(70,00 \%)$ \\
\hline Contusio capitis & $1(0,6 \%)$ & $1(5,6 \%)$ & $0(0 \%)$ \\
\hline Hirnläsion & $5(2,9 \%)$ & $0(0 \%)$ & $0(0 \%)$ \\
\hline Rückenmarkläsion & $9(5,3 \%)$ & $1(5,6 \%)$ & $1(10,00 \%)$ \\
\hline Augenverletzung & $65(38,0 \%)$ & $0(0 \%)$ & $0(0 \%)$ \\
\hline Andere & $4(2,3)$ & $0(0 \%)$ & $1(10,00 \%)$ \\
\hline Total & 171 & 18 & 10 \\
\hline \multicolumn{4}{|l|}{ Körperstelle } \\
\hline Kopf & $85(52,1 \%)$ & $6(42,9 \%)$ & $1(11,11 \%)$ \\
\hline ZNS & $6(3,7 \%)$ & $1(7,1 \%)$ & $1(11,11 \%)$ \\
\hline Gesicht \& Kopf & $13(8,0 \%)$ & $5(35,7 \%)$ & $0(0 \%)$ \\
\hline Auge & $66(52,1 \%)$ & $0(0 \%)$ & $0(0 \%)$ \\
\hline Arm & $16(9,8 \%)$ & $3(21,4 \%)$ & $1(11,11 \%)$ \\
\hline Schulter & $4(2,5 \%)$ & $1(7,1 \%)$ & $0(0 \%)$ \\
\hline Achsel & $1(0,6 \%)$ & $0(0 \%)$ & $0(0 \%)$ \\
\hline Oberarm & $2(1,2 \%)$ & $0(0 \%)$ & $0(0 \%)$ \\
\hline Unterarm & $9(5,5 \%)$ & $2(14,3 \%)$ & $1(11,11 \%)$ \\
\hline Hand & $28(17,2 \%)$ & $2(14,3 \%)$ & $1(11,11 \%)$ \\
\hline Hand & $9(5,5 \%)$ & $1(7,1 \%)$ & $1(11,11 \%)$ \\
\hline Finger & $19(11,7 \%)$ & $1(7,1 \%)$ & $0(0 \%)$ \\
\hline Stamm & $4(2,5 \%)$ & $1(7,1 \%)$ & $5(55,5 \%)$ \\
\hline Nacken & $1(0,6 \%)$ & $0(0 \%)$ & $0(0 \%)$ \\
\hline Brust & $2(1,2 \%)$ & $1(7,1 \%)$ & $2(22,2 \%)$ \\
\hline Rücken & $1(0,6 \%)$ & $0(0 \%)$ & $0(0 \%)$ \\
\hline Wirbelsäule & $0(0 \%)$ & $0(0 \%)$ & $2(22,2 \%)$ \\
\hline Becken & $0(0 \%)$ & $0(0 \%)$ & $1(11,1 \%)$ \\
\hline Bein & $16(9,8 \%)$ & $1(14,3 \%)$ & $0(0 \%)$ \\
\hline Hüfte & $1(0,6 \%)$ & $1(7,1 \%)$ & $0(0 \%)$ \\
\hline Oberschenkel & $5(3,1 \%)$ & $0(0 \%)$ & $0(0 \%)$ \\
\hline Knie & $6(3,7 \%)$ & $0(0 \%)$ & $0(0 \%)$ \\
\hline Unterschenkel & $4(2,5 \%)$ & $1(7,1 \%)$ & $0(0 \%)$ \\
\hline Fuss & $13(8,0 \%)$ & $0(0 \%)$ & $0(0 \%)$ \\
\hline Fuss & $6(3,7 \%)$ & $0(0 \%)$ & $0(0 \%)$ \\
\hline Zehen & $7(4,3 \%)$ & $0(0 \%)$ & $0(0 \%)$ \\
\hline Gastrointestinaltrakt & $0(0 \%)$ & $0(0 \%)$ & $1(11,1 \%)$ \\
\hline Darm & $0(0 \%)$ & $0(0 \%)$ & 1 \\
\hline Unbekannt & $1(0,6 \%)$ & $0(0 \%)$ & $0(0 \%)$ \\
\hline Total & 163 & 14 & 9 \\
\hline
\end{tabular}


en. Männer (54,2 Jahre) waren jünger als Frauen (58,2 Jahre). Alle Verunfallten waren älter als 20 Jahre.

Hinsichtlich der Dringlichkeit der Behandlung wurden sieben $(4,35 \%)$ Patienten als akut lebensbedrohliche Problematik, entsprechend 1, 28 (17,39\%) Patienten als sehr dringende Problematik, entsprechend 2, 115 (71,43\%) Patienten als dringende Problematik, entsprechend 3, neun (5,59\%) Patienten als Standardproblematik, entsprechend 4, und zwei (1,24\%) Patienten als nicht dringende Problematik, entsprechend 5, triagiert (Tab.1). Grundlage der Triage in unserem Notfallzentrum ist das Schweizer Triage System (STS). 25 Verunfallte $(15,5 \%)$ wurden stationär, 129 Verunfallte $(80,1 \%)$ wurden ambulant behandelt, und bei weiteren sieben Verunfallten $(4,3 \%)$ wurden keine Angaben diesbezüglich vorgefunden oder sie wurden aufgrund einer anderen Ursache behandelt. 152 Patienten $(94,4 \%)$ erlitten eine monotraumatische Verletzung, sechs Patienten (3,7\%) kombinierte Verletzungen und drei Patienten (1,9\%) erfüllten die Definition eines Polytraumas mit einem Injury Severity Score grösser 15 (Tab. 1).

\section{Diagnose und betroffene Körperstelle}

Patienten mit monotraumatischen Verletzungen präsentierten sich am häufigsten mit Augenverletzungen (65 Fälle, $38,0 \%$ ) und Hautwunden (51 Fälle, 29,8\%). Bei kombinierten Verletzungen und Polytraumata wurden Frakturen (14 Patienten) am meisten diagnostiziert. Die am häufigsten betroffenen Körperstellen bei monotraumatischen Verletzungen waren Augen (66 Fälle, 52,2\%), Finger (19 Fälle, $11,0 \%)$ und Kopf und Gesicht (13 Fälle, 8,0\%). Bei den kombinierten Verletzungen waren Kopf und Gesicht (5 Fälle) die am meisten betroffenen Körperstellen (Tab. 2, Abb. 2).

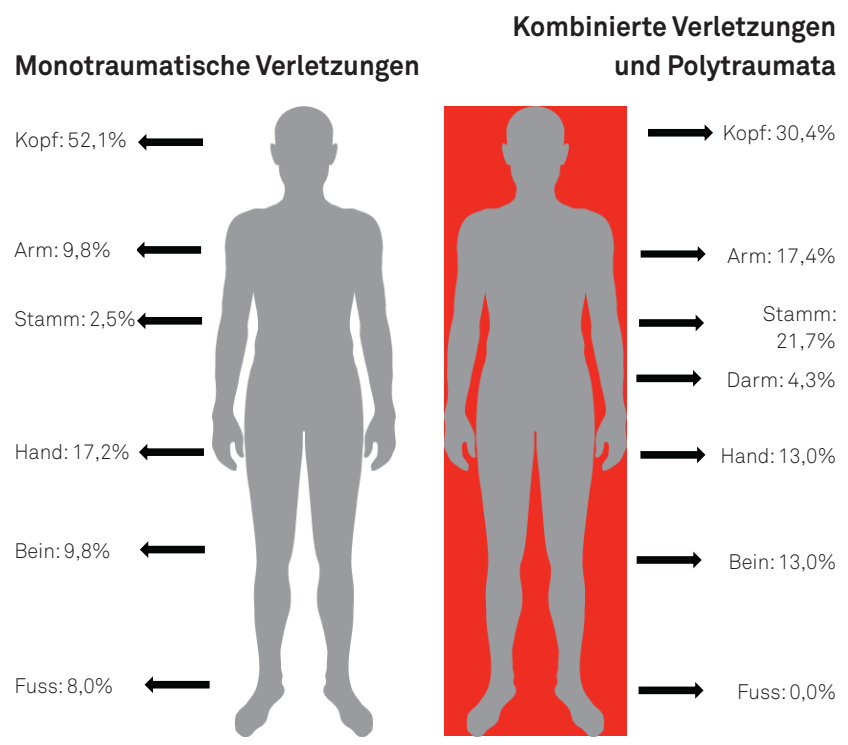

Abbildung 2. Betroffene Körperstelle monotraumatischer, kombinierter und polytraumatischer Verletzungen.

\section{Unfallmechanismen}

Mit 63 Fällen (39,1\%) waren Augenverletzungen die häufigsten Verletzungen, gefolgt von Schnittverletzungen, Stichverletzungen und Schürfwunden mit 36 Fällen $(22,4 \%)$ sowie Sturz- und Stolperereignisse mit 31 Fällen (19,3\%) (Abb. 3A). Augenverletzungen wurden häufig durch Schläge und Hiebe

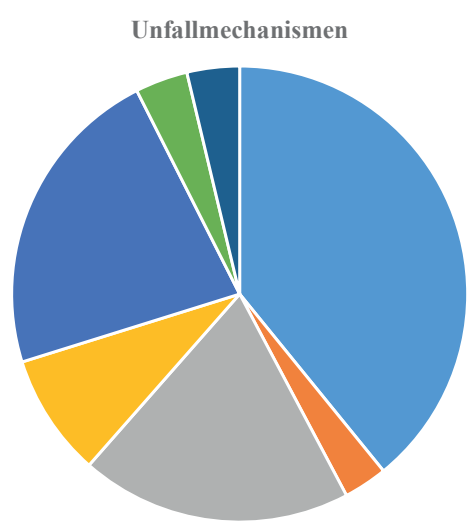

$\begin{array}{ll}\text { - Augenverletzungen } & \text { - Biss- und Stichverletzungen durch Insekten } \\ \text { - Sturzereignis, Stolperereignis } & \| \text { Schlag/Quetschung } \\ \text { - Schnittverletzung, Stichverletzung, Schürfung } & \text { - Undefiniert }\end{array}$

- Atraumatisch

Unfallmechanismen von Augenverletzungen

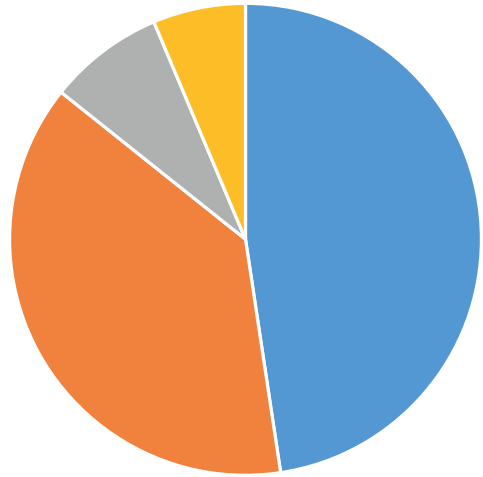

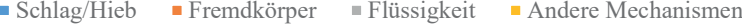

Unfallmechanismen von Sturzereignissen

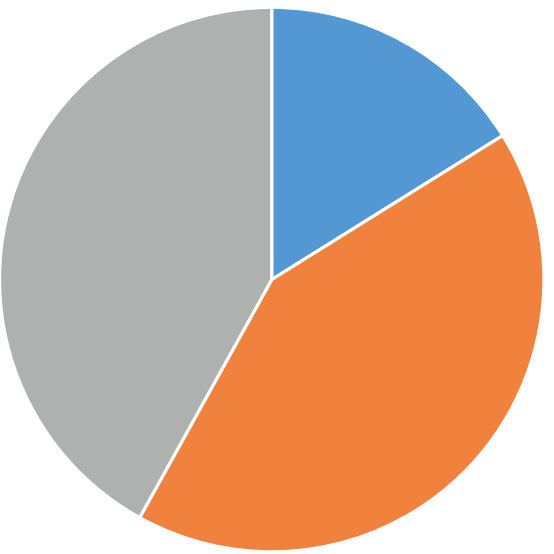

- Stolperereignisse $\quad$ - Stürze aus Körperhöhe $\quad$ - Stürze über Körperhöhe

Abbildung 3. Unfallmechanismen (A), Unfallmechanismen von Augenverletzungen (B) und Unfallmechanismen von Stürzen (C). 
(30 Fälle, 47,6\%) verursacht, gefolgt von losen Fremdkörpern in 24 Fällen (38,1\%), Flüssigkeiten in fünf Fällen (7,9\%) und anderen Mechanismen in vier Fällen (6,3\%) (Abb. 3B). Stürze aus Körperhöhe ereigneten sich ebenso häufig wie Stürze aus mehr als Körperhöhe (in beiden Kategorien 13 Fälle, 41,9\%). Bei weiteren fünf Fällen (16,1\%) handelte es sich um Stolperereignisse oder stolperähnliche Mechanismen. Stürze aus mehr als Körperhöhe erfolgten meistens von Mauern (6 Fälle) (Abb. 3C).

\section{Unfallverursachende Objekte}

Die meisten Unfälle wurden durch organische Materialien wie Pflanzen, Holz oder Erde verursacht. (58 Fälle, 36,0 \%). Pflanzen führten durch ihre Äste (27 Fälle), Dornen (7 Fälle), Blätter (6 Fälle) oder Säfte (4 Fälle) zu Verletzungen. Weitere 23 Fälle $(14,3 \%)$ ereigneten sich durch die fehlerhafte und/oder nachlässige Anwendung von Gartenwerkzeugen. In 14 dieser 23 Fälle waren es Schneidwerkzeuge (Abb. 4).

\section{Diskussion}

Diese Studie beschreibt als erste Gartenarbeitsunfälle in der Schweiz. Mehrere Studien beschreiben die positiven
Auswirkungen des Gärtnerns auf die Gesundheit [7, 8 14]. Die Korrelation zwischen Gartenarbeit und Gesundheit wurde in unterschiedlichen Aspekten erforscht, wie beispielsweise Linderung von Depressionen und Stress, Gewichtsreduktion, Zunahme der Lebensqualität, interpersonale Kontakte sowie mehr Körperaktivität [8,14].

Freizeit-Gartenarbeitsunfälle erlitten vorwiegend Erwachsene mittleren Alters. Dabei stellten sich mehr männliche Patienten auf dem Notfall vor. Bei Konsultation präsentierten sich die Patienten mit vorwiegend monotraumatischen Verletzungen, die ambulant behandelt werden konnten. Diese Datenlage führt zu der Annahme, dass es sich bei einem Grossteil der Vorstellungen um Bagatellunfälle handelte. Patienten mit monotraumatischen Verletzungen beklagten vorwiegend Augenverletzungen und Hautwunden. Patienten mit kombinierten Verletzungen und Polytraumen präsentierten sich am häufigsten mit Frakturen. Zudem handelte es sich grösstenteils um Verletzungen an Augen, Finger, Kopf und Gesicht. Bei Augenverletzungen handelte es sich um den häufigsten Unfallmechanismus, gefolgt von Schnittverletzungen, Stichverletzungen und Schürfwunden sowie Sturz- und Stolperereignisse.

Obschon sich jährlich 16000 Nichtberufsunfälle durch Gartenarbeit in der Schweiz ereignen, ist Gärtnern eine geeignete Freizeitaktivität, um körperliche Aktivität und Zeit in der Natur zu verbinden. Daher sollten die positiven Auswirkungen des Gärtnerns auf Körper und Geist nicht unterschätzt werden $[8,9,12-14]$.

\section{Unfallverursachende Objekte}

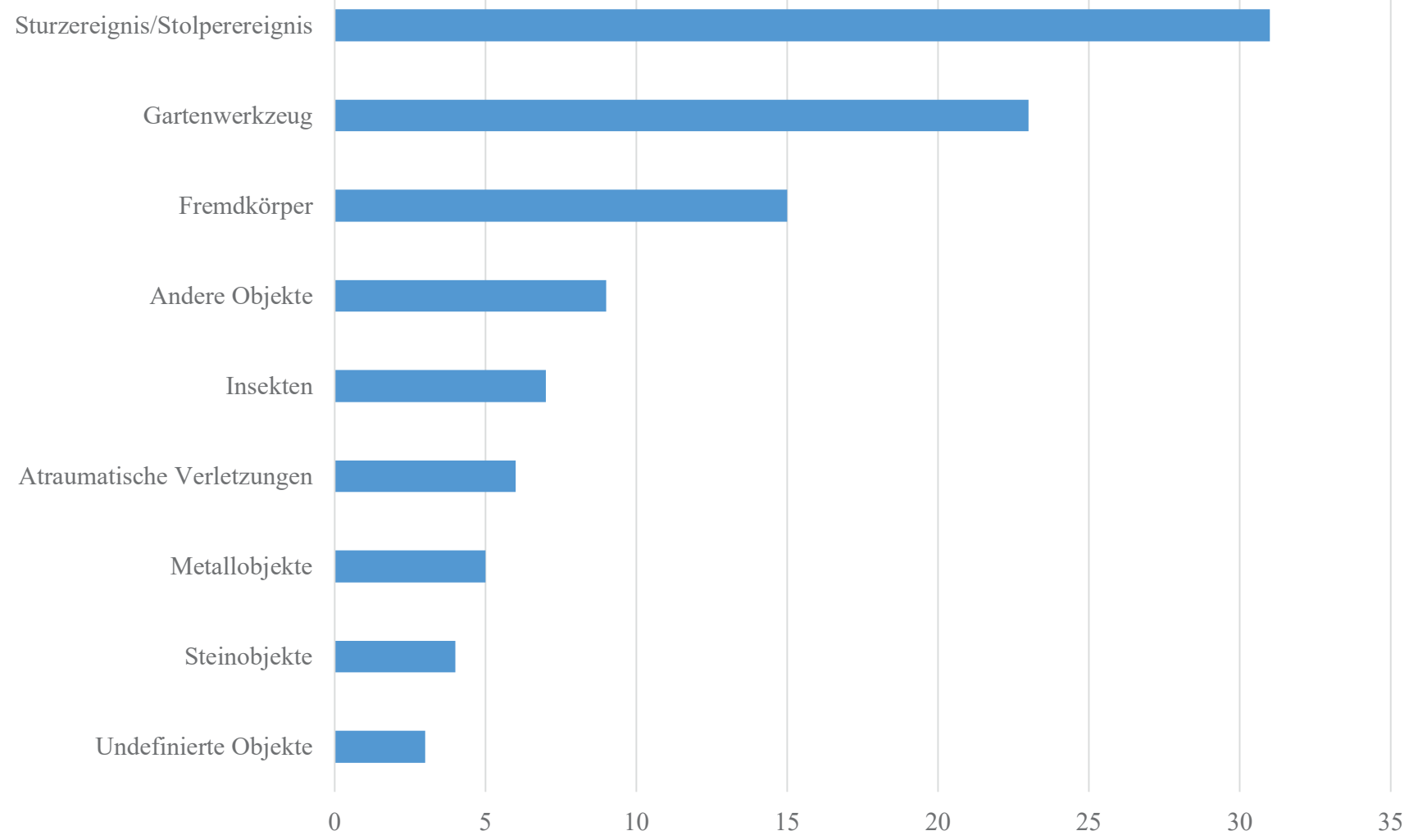

Abbildung 4. Unfallverursachende Objekte. 


\section{Prävention}

Die Analyse von Gartenarbeitsunfällen zeigte die Risiken des Gärtnerns auf [10, 11]. Augenverletzungen, Schnittwunden, Strichverletzungen und Schürfwunden, sowie Sturz- und Stolperereignisse sind die häufigsten Unfallmechanismen. Verletzungen waren vorwiegend an exponierten Körperstellen wie Kopf und Augen sowie Händen vorzufinden. Mit einfachen Präventionsmassnahmen könnten diese Verletzungen verhindert werden. In einer retrospektiven Analyse zeigte sich, dass ein Grossteil der Augenverletzungen durch Schutzbrillen hätte verhindert werden können [18, 19], die Augen vor Hieben und Schlägen, Fremdkörpern und Flüssigkeiten schützen [20]. Hautverletzungen können durch das Tragen angemessener Kleidung vermieden werden; Handschuhe sind unentbehrlich im Kontakt mit Pflanzen oder beim Gebrauch von Werkzeugen. Handschuhe, die einen mechanischen Schutz und isolierende Eigenschaft bieten, eignen sich zum Gärtnern am besten [21, 22]. Obschon Insektenstiche und Zeckenbisse lediglich eine kleine Fraktion der Verletzungen ausmachen, sollte ein angemessener Schutz diesbezüglich nicht vernachlässigt werden. Das Tragen langärmliger Kleidung und der Gebrauch von Insektenspray kann Stiche und Bisse verhindern. Zudem sollte nach Aufenthalt in einem zeckenreichen Gebiet eine körperliche Untersuchung erfolgen, um eventuelle Zecken schnellstmöglich zu entfernen und somit das Infektionsrisiko zu reduzieren [23]. Sturzereignisse sind die häufigste Ursache für Unfälle im häuslichen Umfeld und in der Freizeit [24]. Gutes Schuhwerk gewährleistet eine sichere Bodenhaftung und Rutschfestigkeit. Ordnung im Garten ist ebenfalls eine wichtige präventive Massnahme, wodurch Stolperfallen beseitigt werden [24]. Zudem hilft es, ein Vorgehen zu planen. Vorausschauendes Handeln enthüllt öfters versteckte Gefahren [25-28].

\section{Einschränkungen}

Diese retrospektive Analyse hat einige Einschränkungen. Die Datenbank Ecare detektierte lediglich Gartenarbeitsunfälle des Universitären Notfallzentrums Bern. Daher wurden Verunfallte nach Gartenarbeitsunfällen, die sich in anderen Notfallzentren oder bei einem Hausarzt vorstellten, nicht erfasst, wodurch sich die niedrigen Fallzahlen und somit eine reduzierte Validität dieser Studie erklären lässt. Die Erkenntnisse dieser retrospektiven Analyse könnten daher in Bezug auf Gartenarbeitsunfälle in der Schweiz nicht repräsentativ sein. Diese Studie verwendete grösstenteils Berichte in narrativer Form, dadurch ist von einer teils ungenauen Berichterstattung bezüglich der Anamnese und der Befunde auszugehen, was mit Selektions- und Interpretationsfehlern einhergehen kann. Trotz ihrer Einschränkungen sind auch Stärken dieser Studie zu nennen, wie das definierte Ausschlussverfahren, das zu einem niedrigen Selektionsbias führt. Aus-

\section{Key messages}

- Bei den Gartenunfällen handelte sich vor allem um monotraumatische Verletzungen bei mittelalten $\mathrm{Pa}$ tienten, die ambulant behandelt werden konnten.

- Patienten beklagten hauptsächlich Augen- und Weichteilverletzungen (Finger, Kopf und Gesicht).

- Viele Gartenunfälle hätten mittels einfacher präventiver Massnahmen verhindert werden können.

serdem sind die demografischen Daten sehr genau erfasst. Obschon die Generabilität dieser Analyse limitiert ist, sind die erhaltenen Erkenntnisse mit anderen Studien vereinbar [29-32].

\section{Zusammenfassung}

In dieser retrospektiven Analyse erlitten vorwiegend Erwachsene mittleren Alters Gartenarbeitsunfälle. Es präsentierten sich mehr männliche Verunfallte in unserem Notfallzentrum. Dabei handelte es sich vorwiegend um monotraumatische Verletzungen, die ambulant behandelt werden können. Die Verunfallten verletzen sich am häufigsten an exponierten Körperstellen wie Augen, Finger, Kopf und Gesicht, beklagt wurden hauptsächlich Augenund Weichteilverletzungen. Die meisten Unfälle wurden durch organische Materialien verursacht oder durch die fehlerhafte und/oder nachlässige Anwendung von Gartenwerkzeugen. Zudem gab es insgesamt drei Polytraumen bei Gartenarbeit als Freizeitaktivität.

Diese Analyse präsentiert die möglichen Gefahren des Gärtnerns. Ein Grossteil dieser Unfälle hätte mittels einfacher präventiver Massnahmen verhindert werden können.

Es werden weitere Studien benötigt, um Gartenarbeitsunfälle zu erforschen und effiziente Präventionsstrategien zu entwickeln.

\section{Bibliografie}

1. Detweiler MB, Sharma T, Detweiler JG, et al.: What is the evidence to support the use of therapeutic gardens for the elderly? Psychiatry Investig 2012; 9: 100-110. doi: 10.4306/ pi.2012.9.2.100. PubMed PMID: PMC3372556.

2. Ashe MC, Miller WC, Eng JJ, Noreau L, Physical Activity and Chronic Conditions Research Team: Older adults, chronic disease and leisure-time physical activity. Gerontology 2009; 55: 64-72. doi: 10.1159/000141518. PubMed PMID: 18566534; PubMed Central PMCID: PMCPMC3167824.

3. Statistik Bf. 2003-2006, updated 09.01.2013. https://www. bfu.ch/sites/assets/Shop/bfu_2.097.01_bfu-Sicherheitsdos sierNr.09\%E2\%80\%93HausundFreizeit.pdf; letzter Zugriff: 22.05.2019

4. Michel FI Bochud Y: Haus und Freizeit. Unfall-, Risiko- und Interventionsanalyse. 2012. https://www.bfu.ch/sites/assets/Shop/ bfu_2.097.01_bfu-Sicherheitsdossier Nr. 09\%E2\%80\%93 Haus und Freizeit.pdf; letzter Zugriff: 22.05.2019. 
5. Chen AJ, Kim JG, Linakis JG, Mello MJ, Greenberg PB: Eye injuries in the elderly from consumer products in the United States: 2001-2007. Graefes Arch Clin Exp Ophthalmol 2013; 251: 645-651. doi: 10.1007/s00417-012-2004-x. PubMed PMID: 22527310

6. Bhogal G, Tomlins PJ, Murray PI: Penetrating ocular injuries in the home. J Public Health (Oxf) 2007; 29: 72-74. doi: 10.1093/ pubmed/fdl074. PubMed PMID: 17090631.

7. Nicklett EJ, Anderson LA, Yen IH: Gardening activities and physical health among older adults: A review of the evidence. $J \mathrm{Appl}$ Gerontol 2016; 35: 678-690. doi: 10.1177/0733464814563608. PubMed PMID: PMC4469628.

8. York M, Wiseman T: Gardening as an occupation: A critical review. Br J Occupat Ther 2012; 75: 76-84. doi: 10.4276/030802212X1 3286281651072.

9. Husk K, Lovell R, Garside R: Prescribing gardening and conservation activities for health and wellbeing in older people. Maturitas 2018;110:A1-A2. doi:10.1016/j.maturitas.2017.12.013. PubMed PMID: 29279141.

10. Panatto D, Gasparini R, Vitale A, et al.: Survey of domestic accidents in the elderly in the Province of Genoa (northern Italy). J Prev Med Hyg 2009; 50: 53-57. PubMed PMID: 19771761.

11. Parkkari J, Kannus P, Natri A, et al.: Active living and injury risk. IntJ Sports Med 2004:25:209-216. doi:10.1055/s-2004-819935. PubMed PMID: 15088246.

12. Hassan A, Qibing C, Tao J: Physiological and psychological effects of gardening activity in older adults. Geriatr Gerontol Int 2018. doi: 10.1111/ggi.13327. PubMed PMID: 29626378.

13. Tournier I, Postal V: [An integrative model of the psychological benefits of gardening in older adults]. Geriatr Psychol Neuropsychiatr Vieil 2014; 12: 424-431. doi: 10.1684/ pnv.2014.0498. PubMed PMID: 25515907.

14. Soga M, Gaston KJ, Yamaura Y: Gardening is beneficial for health: A meta-analysis. Prev Med Rep 2017; 5: 92-99. doi: 10.1016/j.pmedr.2016.11.007. PubMed PMID: PMC5153451.

15. Inselspital Bern, Universitäres Notfallzentrum. Über uns. 2017. http://www.notfallzentrum.insel.ch/de/ueber-uns/; letzter Zugriff: 22.05 .2019

16. Koordination Schweiz: Online-Handbuch. Unfallbegriff. 2018. https://www.koordination.ch/online-handbuch/atsg/unfallbegriff/; letzter Zugriff: 22.05.2019.

17. Koordination Schweiz: Online-Handbuch. Körperschädigung. 2018. https://www.koordination.ch/online-handbuch/ uvg/koerperschaedigung/; letzter Zugriff: 22.05.2019.

18. Patel D: Preventing eye injuries. Community Eye Health 2015; 28: 51. PubMed PMID: 26989313; PubMed Central PMCID: PMCPMC4790163.

19. Eye protection. Farm and Ranch extension in Safety and Health (FReSH) Community of Practice. 2013. http://www. extension.org/pages/66976/eye-protection-for-agriculturalworkers; letzter Zugriff: 22.05.2019.

20. Suva: Augenschutz. Arbeit ohne Sehverlust mit Persönlicher Schutzausrüstung. 2018. https://www.suva.ch/material/ factsheets/augenschutz; letzter Zugriff: 22.05.2019.

21. Suva: Handschutz, Armschutz und Hautschutz. Das Wichtigste über Persönliche Schutzausrüstungen für Arm und Hand.
2018. https://www.suva.ch/material/factsheets/handschutz armschutz und hautschutz; letzter Zugriff: 22.05.2019.

22. 2mains.ch: Schutzhandschuhe für Gärtner, Gartenbauer, Weinbauer 2018. http://www.2mains.ch/de/professions/59; letzter Zugriff: 22.05.2019.

23. Ineichen F: DHR. Vorsicht, Zecken! 2016. https://www.suva. $\mathrm{ch} / \mathrm{material} /$ dokumentationen/vorsicht-zecken-44051. d-6026-6026; letzter Zugriff: 22.05.2019.

24. Suva: Ratgeber Unfallverhütung. Im und ums Haus. Sturzprävention. 2018. https://www.bfu.ch/de/ratgeber/ratgeberunfallverh\%C3\%BCtung/im-und-ums-haus/st\%C3 \%BCrze/ st\%C3\%BCrze/stuerze; letzter Zugriff: 22.05.2019.

25. Schweizer Familiengärtner-Verband. 2010. Dokumentation. Im Garten sicher arbeiten. http://www.familiengaertner.ch/de/ dienstleistungen/dokumentationen; letzer Zugriff: 28.05.2019

26. Unfallverhütung: Bf. bfu-Sicherheitstipp «Gartenarbeiten» 2015. https://www.bfu.ch/de/Documents/05_Die_bfu/13_ Sicherheitstipps/1509/Sicherheitstipp-1509.pdf; letzter Zugriff: 22.05.2019.

27. Unfallverhütung Bf: Sicherheitstipps. Gartenarbeit im Frühling. 2018. https://www.bfu.ch/de/Sicherheitstipps/gartenarbeitenim-fruehling; Letzter Zugriff: 22.05.2019.

28. Unfallverhütung Bf: Sicherheitstipps. bfu-Tipps für Gartenarbeiten ohne Verletzungen 2014. 2014. Available from: https:// www.bfu.ch/de/Documents/05_Die_bfu/13_Sicherheits tipps/1409/Sicherheitstipp-1409.pdf; letzter Zugriff: 22.05.2019.

29. Powell KE, Heath GW, Kresnow MJ, Sacks JJ, Branche CM: Injury rates from walking, gardening, weightlifting, outdoor bicycling, and aerobics. Med Sci Sports Exerc 1998; 30: $1246-$ 1249. PubMed PMID: 9710864.

30. McGwin G Jr, Owsley C: Incidence of emergency departmenttreated eye injury in the United States. Arch Ophthalmol 2005; 123: 662-666. doi: 10.1001/archopht.123.5.662.

31. Watson DS, Shields BJ, Smith GA: Trimming- and pruningrelated injuries in the United States, 1990 to 2007. J Trauma Acute Care Surg 2012; 72: 257-262. doi: 10.1097/ TA.0b013e318239ceed

32. Majori S, Ricci G, Capretta F, Rocca G, Baldovin T, Buonocore F: Epidemiology of domestic injuries. A survey in an emergency department in North-East Italy. J Prev Med Hyg 2009; 50:164169.

Manuskript eingereicht: 23.04.2019

Manuskript akzeptiert: 21.05.2019

Interessenskonflikt: Die Autoren erklären, dass keine Interessenskonflikte bestehen

\section{Dr. Jolanta Klukowska-Rötzler}

Notfallzentrum, Universitätsspital Bern 3010 Bern

jolanta.klukowska-roetzler@insel.ch 